\title{
Factors regulating ventricular rates during atrial flutter and fibrillation in pre-excitation (Wolff-Parkinson- White) syndrome
}

\author{
Agustin Castellanos, Jr., Robert J. Myerburg, Karen Craparo, Benjamin Befeler, \\ and Abdul S. Agha \\ From the Medical Service Veterans Administration Hospital, and the Department of Medicine, Division of \\ Cardiology, University of Miami School of Medicine, Miami, Florida, U.S.A.
}

Premature atrial stimulation and His bundle recordings were performed in 6 patients with pre-excitation (WPW) syndrome. The ventricular rates during bursts of atrial flutter and atrial fibrillation triggered by the earliest impulses depended on the functional properties of the accessory pathway. In 3 patients in whom the effective refractory period of the accessory pathway was longer than that of the normal AV pathway the ventricular rates did not exceed $210 / m i n ;$ the majority of the supraventricular impulses reached the ventricles through the normal $A V$ pathway. In 3 other patients in whom the effective refractory period of the accessory pathway was very short (less than $220 \mathrm{msec}$ ) the ventricular rates were as high as 310/min; the majority of the impulses reached the ventricles through the accessory pathway. Concealed $A V$ conduction occurred in both pathways producing $R R$ pauses well in excess of the duration of the corresponding effective refractory periods. These tachyarrhythmias were due to stimulation during the atrial vulnerable period, not to a reciprocating mechanism involving two separate anatomical $A V$ communications.

Intracardiac recordings were necessary to evaluate the $A V$ conduction patterns as well as the nature of the wide $Q R S$ complexes seen during the tachyarrhythmias.

Factors regulating the ventricular rates during atrial flutter and fibrillation in patients with pre-excitation (WPW) syndrome are not well known. Previous studies from our department suggested that the AV conduction patterns occurring during these tachyarrhythmias depended on the duration of the effective refractory period of the accessory pathway and on its relation to that of the normal pathway (Castellanos et al., 1973).

The present communication corroborates these assumptions.

\section{Subjects and methods}

The technique for obtaining His bundle recordings used in our department has been discussed previously (Castellanos and Castillo, 1972; Castellanos et al., 1973). After consent was obtained from the patient and discontinuing all drugs for at least three days, premature atrial stimulation and His bundle recordings were performed during a basic paced atrial drive in 6 patients with the type of pre-excitation (WPW) syndrome in which the forward $H$ deflection was inscribed within the $Q R S$ complex. During the course of the study bursts of atrial flutter and Received 5 February 1973. fibrillation were triggered by some of the most premature atrial stimuli. The analysis of these arrhythmias constitutes the basis for this report.

The effective refractory period of the accessory AV pathway was given by the longest $A_{1}-A_{2}$ interval at which $A_{2}$ failed to produce a $Q R S$ complex with a delta wave (Castellanos et al., 1973). The effective refractory period of the normal AV pathway was given by the longest $A_{1}-A_{2}$ at which $A_{2}$ failed to produce a 'fusion' or 'combination' QRS complex (Castellanos et al., 1973).

\section{Results}

There were 3 patients in whom the effective refractory period of the accessory pathway was longer than that of the AV node (Table I). At long coupling intervals AV conduction occurred through the accessory pathway, but once a given interval was reached propagation to the ventricles occurred exclusively via the normal AV pathway. Very premature stimuli triggered runs of atrial flutter and fibrillation during which nearly all impulses reached the ventricles exclusively through the normal AV pathway. Occasional fusion beats were noted. Exclusive accessory pathway conduction was 
TABLE I Data on 3 patients in whom effective refractory period of accessory pathway was longer than that of normal pathway ${ }^{\star}$

\begin{tabular}{lllll}
\hline $\begin{array}{l}\text { Case } \\
\text { No. }\end{array}$ & $\begin{array}{l}\text { WPW } \\
\text { type }\end{array}$ & \multicolumn{3}{c}{$\begin{array}{c}\text { Effective refractory } \\
\text { period } \\
\text { Accessory }\end{array}$} \\
& & $\begin{array}{l}\text { Normal } \\
\text { pathway }\end{array}$ & $\begin{array}{l}\text { Driving cycle length of } \\
\text { high rt. atrium }\end{array}$ \\
\hline it & B & 350 & 265 & 640 \\
2 & A & 410 & 310 & 680 \\
3 & A & 360 & 315 & 600 \\
\hline
\end{tabular}

$\star$ All values in milliseconds.

† For further information see Case $I$ of the article by Castellanos et al. (1973).

rare in these cases (Fig. I). The ventricular rates did not exceed $210 / \mathrm{min}$. These arrhythmias were of short duration and disappeared spontaneously.

In 3 other patients (Table 2) the effective refractory period of the accessory pathway was very short (less than $220 \mathrm{msec}$ ). Its exact duration could not be determined in Cases 4 and 6 because of the occurrence of persistent tachyarrhythmias, and in Case 5 because the atria became effectively refractory while conduction was still occurring through the accessory pathway. Neither could the effective refractory period of the normal AV pathway be measured with
TABLE 2 Data on 3 patients in whom effective refractory period of accessory pathway was extremely short, presumably shorter than that of normal pathway*

$\begin{array}{llcll}\begin{array}{l}\text { Case } \\ \text { No. }\end{array} & \begin{array}{l}\text { WPW } \\ \text { type }\end{array} & \begin{array}{c}\text { Effective refractory } \\ \text { period } \\ \text { Accessory }\end{array} & \begin{array}{l}\text { Normal } \\ \text { high ring cycle length of } \\ \text { pathwayt }\end{array} \\ & & \text { pathway }\end{array}$

$\star$ All values in milliseconds.

+ Effective refractory period shorter than these values. $¥$ For further information see Case 2 of the article by Befeler et al. (1973).

$\$$ For further information see Case 2 of the article by Castellanos et al. (1973).

** For further information see Case 4 of the article by Castellanos et al. (1973); and Case 3 of the article by Castillo et al. (1973).

accuracy since the coupling interval at which a fusion QRS complex failed to occur could not be determined. This was due to the fact that at short coupling intervals the supraventricular impulses reached the ventricles while the latter were incompletely recovered, therefore causing some

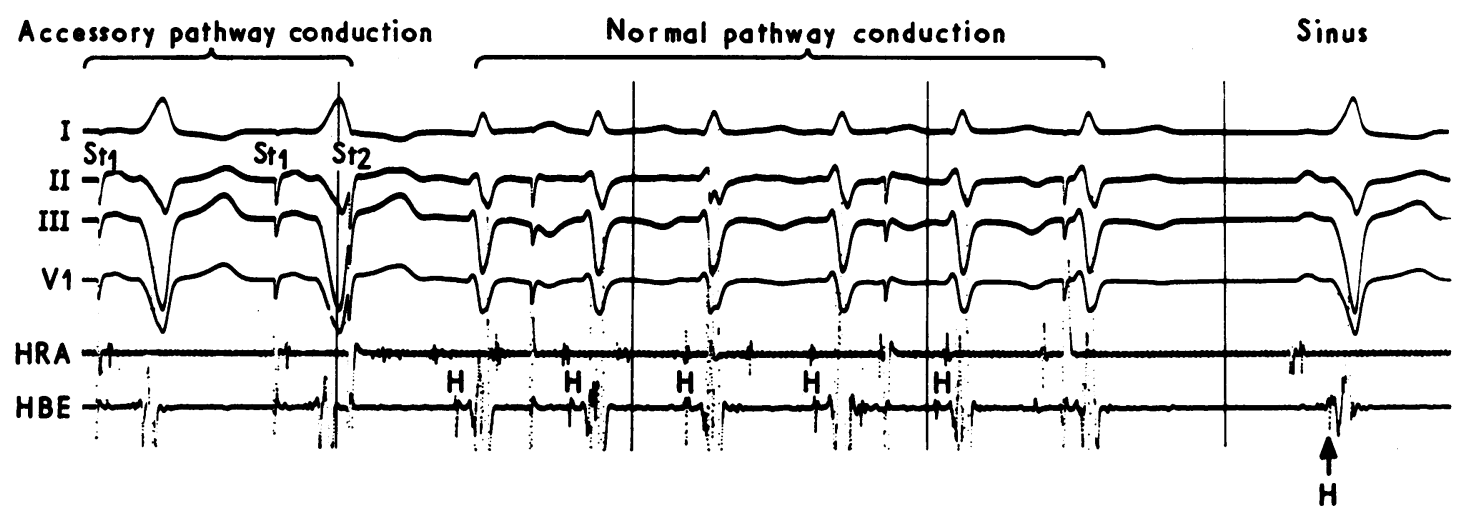

FIG. I (Case I) Short run of atrial flutter (?) in a patient with WPW type $B$ in which the effective refractory period of the accessory $A V$ pathway exceeded that of the normal $A V$ pathway. Driven impulses (StI) were conducted exclusively or predominantly through the accessory pathway (first two $Q R S$ complexes). The forward $H$ deflection was lost within the ventricular electrogram of the HBE lead. A premature stimulus (St2) delivered at a coupling interval of 250 msec triggered a short run of atrial fibrillation or atrial flutter which subsided spontaneously. During the tachyarrhythmia AV conduction occurred exclusively through the normal pathway as determined by the presence of an $H$ deflection in front of the $Q R S$ complexes without a delta wave and a left anterior hemiblock morphology. The HV interval was of normal duration. The last beat was a fusion complex produced by a $P$ wave of sinus origin. Note that the forward $H$ deflection was inscribed after the onset of depolarization. HRA=bipolar electrogram from the high right atrium. HBE $=$ His bundle electrogram. In this and all figures (except Fig. 2) paper speed and time markers are $100 \mathrm{~mm} / \mathrm{sec}$ and I sec, respectively. 
distortion of the ventricular complexes. It was not possible to determine the coupling interval at which a fusion beat (resulting from AV conduction through both pathways) ended and when aberration due to incomplete ventricular recovery started. However it is unusual for the effective refractory period of the normal pathway to be less than $220 \mathrm{msec}$ (Wit et al., 1970).

In these 3 patients (Cases 4,5 , and 6 ) the ventricular rates during the arrhythmias were as high as $3 \mathrm{ro} / \mathrm{min}$. Nearly all supraventricular impulses reached the ventricles exclusively, or predominantly, through the accessory pathway. The resulting QRS complexes either had a 'pure' WPW contour, or a WPW morphology distorted by incomplete ventricular recovery. Fusion beats were also noted. Occasional impulses propagated exclusively through the normal AV pathway.

All 3 patients showed periods in which the RR intervals were as long as $600 \mathrm{msec}$. During these pauses AV conduction did not occur through either pathway, presumably because of concealed conduction in both structures.

The atrial arrhythmias in Cases 4,5 , and 6 did not disappear spontaneously. They required transthoracic electrical conversion for restoration of sinus rhythm.

\section{Discussion}

Mechanisms of atrial flutter and fibrillation in pre-excitation (WPW) syndrome

Tachyarrhythmias occurring in patients with WPW syndrome can be due to several mechanisms. For

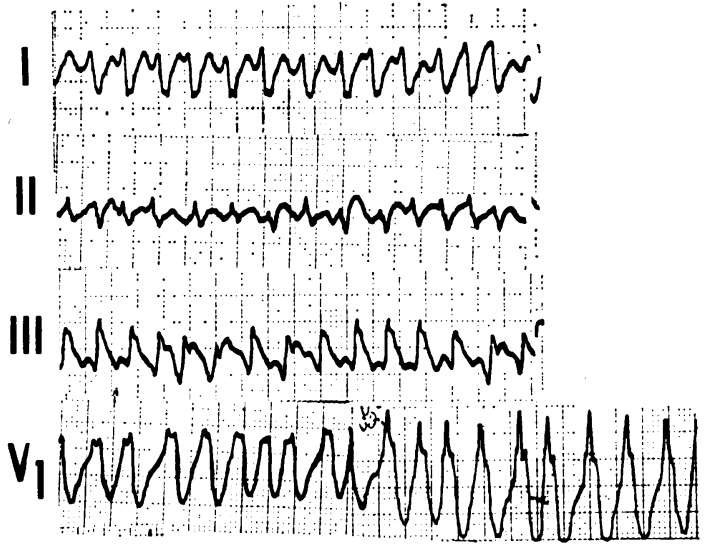

FIG. 2 (Case 5). Persistent atrial fibrillation in a patient with WPW type $A$ in which the effective refractory period of the accessory pathway was shorter than 190 msec. The supraventricular impulses reached the ventricles exclusively, or predominantly, through the accessory pathway. The ventricular rates were as high as $310 \times$ min.

instance, in 1964 Scherf and Cohen suggested that a reciprocating rhythm involving two separate anatomical pathways (Kent bundle and normal AV junction) could not explain the occurrence of atrial fibrillation.

The newer techniques of atrial pacing and His bundle recordings have shown that premature stimuli delivered early in the cycle (soon after the

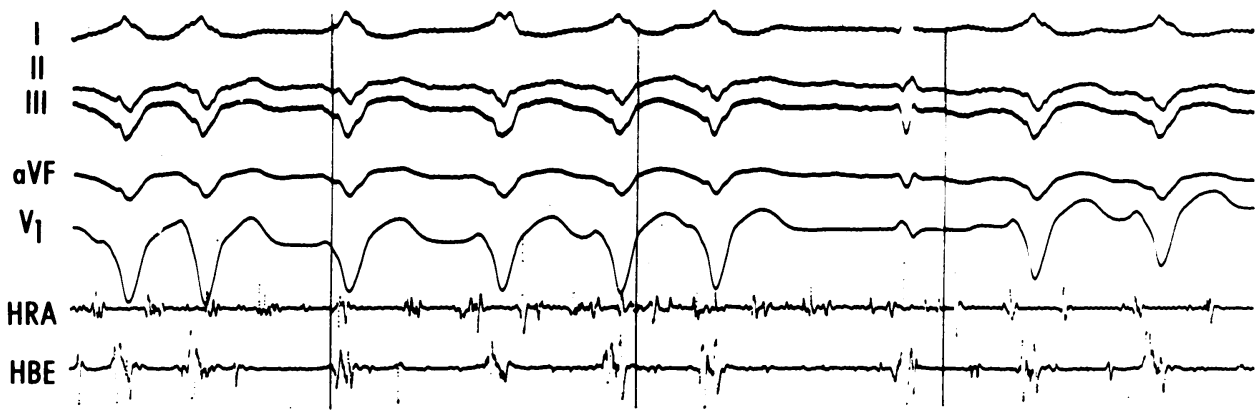

FIG. 3 (Case 4). Persistent atrial fibrillation in a patient with WPW type $B$ in which the effective refactory period of the accessory pathway was shorter than 220 msec.

All supraventricular impulses (except one) reached the ventricles exclusively through the accessory pathway. The third $Q R S$ complex from the end of the strip was conducted to the ventricles through the normal pathway with some degree of right bundle-branch block. The relatively long $R R$ interval (630 msec) preceding this ventricular complex occurred because of the concealed penetration (of supraventricular impulses) into both pathways. Though some of the sharper deflections recorded by the His bundle electrogram lead might have been $H$ deflections the majority were atrial in origin. The fastest ventricular rate recorded in this patient was $270 \times$ min. 
end of the atrial effective refractory period) could initiate runs of reciprocating tachycardia as well as of flutter or fibrillation (Castillo and Castellanos, 1970). The latter were induced by the electrical stimuli falling in the vulnerable period of the atria. Whereas reciprocating tachycardia was terminated by vagal manoeuvres and properly timed electrical stimuli (Castillo and Castellanos, I970) atrial fibrillation disappeared either spontaneously, or required electrical conversion. In earlier studies it was believed that persistence of the latter arrhythmia was related to the presence of coexisting atrial disease (Castillo and Castellanos, 1970). Nevertheless the events in Cases 4 to 6 suggested that they might persist in patients without clinically or haemodynamically detectable atrial diseases provided that the effective refractory period of the accessory pathway is very short (Table 2 and Fig. 2-6). This is in contrast to what occurs in the cases in which the effective refractory period of the accessory pathway is relatively long (Table I and Fig. I). The reasons for this behaviour of the fibrillating atria are not clear.

AV conduction patterns and ventricular rates during atrial flutter and fibrillation in preexcitation (WPW) syndrome

Several authors have observed that the ventricular rates in atrial flutter and fibrillation can vary from

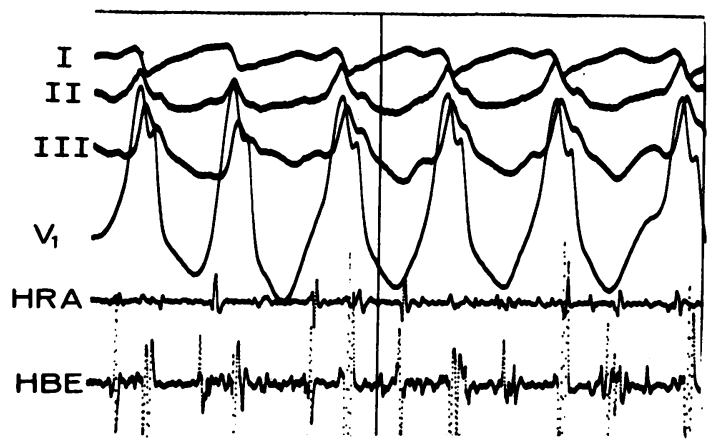

FIG. 4 (Case 6). Atrial fibrillation in a patient with WPW type $A$ in which the effective refractory period of the accessory pathway was shorter than 220 msec. Information regarding this patient (specifically the QRS morphology resulting from exclusive accessory pathway conduction) can be found in previous articles (Castellanos et al., 1973; Castillo et al., 1973).

All supraventricular impulses reached the ventricles exclusively through the accessory pathway. The distortion created by the fibrillating atria in the His bundle electrogram lead made (as in Fig. 3) the identification of $\boldsymbol{H}$ deflection impossible. very fast (pseudoventricular tachycardia [Herrmann et al., 1957]) to relatively slow (between 90 and 120 msec) (Katz and Pick, 1956; Laham, 1969).

Conduction through both pathways has been known to alternate but the reasons why the rates could vary so much have been a matter of debate and speculation. The present communication and a previous study (Castellanos et al., 1973) suggested that ventricular rate depended on the duration of the effective refractory period of the accessory pathway. When it was longer than that of the normal AV pathway (and AV nodal block was not present) the majority of the impulses were transmitted across the normal AV pathway through the
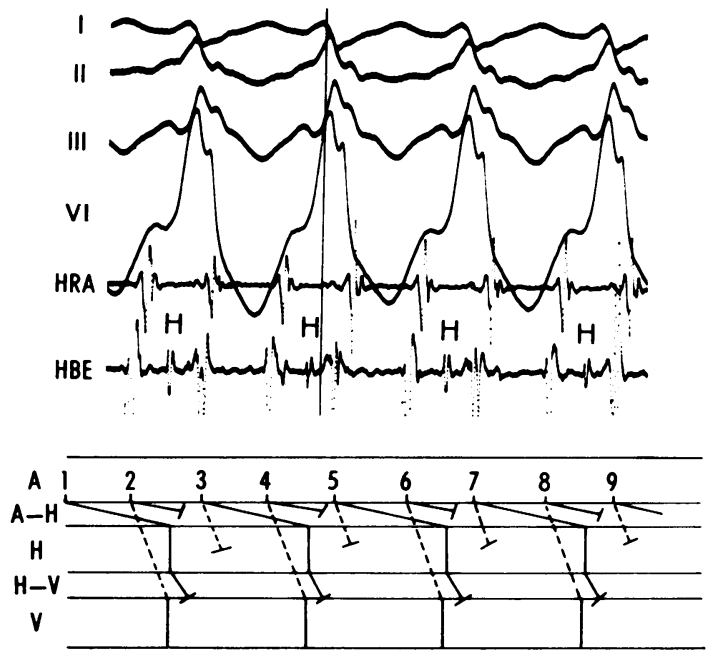

FIG. 5 (Case 6). Atrial flutter with 2:I AV conduction (to the ventricles) exclusively through the accessory pathway. Note that all $\mathrm{H}$ deflections are inscribed after the onset of ventricular depolarization. The atrial rate was $300 \times$ min. Numbers at the $A$ level indicate consecutive low right atrial impulses.

Broken oblique lines extending from $A$ to $V$ represent conduction through the accessory pathway. Solid lines at the AH level indicate propagation through the $A V$ node. The tracings show 2:I $A V$ conduction through the accessory pathway since $A_{2}, A_{4}, A_{6}$, and $A_{8}$ were conducted, while $A_{1}$ (not shown) $A_{3}, A_{5}, A_{7}$, and $A_{9}$ failed to do so, either because the accessory pathway was effectively refractory or because the impulses 'decremented' through it. In addition there was $2: 1$ $A V$ block at the $A V$ node. $A_{1}$ (not shown), $A_{3}, A_{5}$, and $A_{7}$ activate His bundle (thereafter being unable to depolarize the ventricles due to the ventricular refractoriness created by the impulses traversing the accessory pathway). However $A_{2}, A_{4}, A_{6}$, and $A_{8}$ were not followed by $H$ deflections indicating that they were blocked within the $A V$ node. 

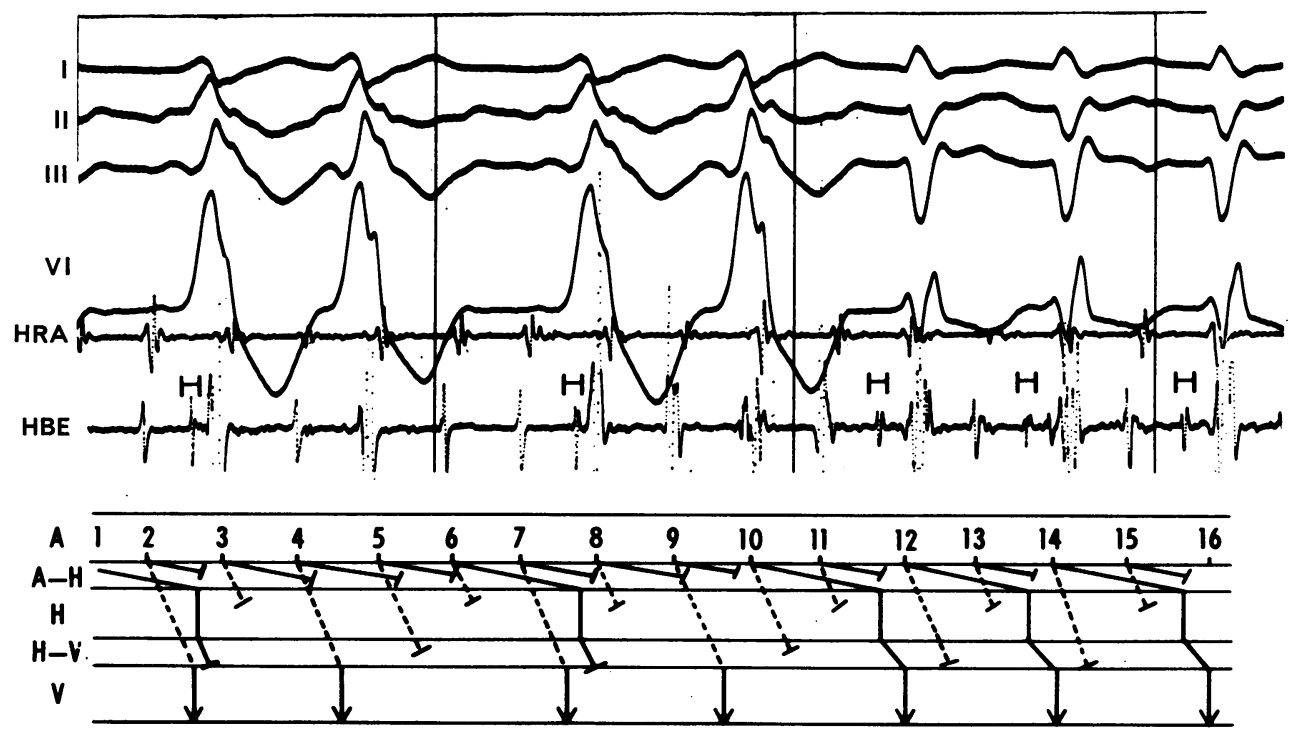

FIG. 6 (Case 6). Atrial flutter with varying AV conduction through both pathways. The first four $Q R S$ complexes resulted from exclusive accessory pathway conduction. During this part of the tracing there was $2: 1$ and $3: I$ accessory pathway conduction alternating with $5: 1$ and $4: I$ conduction between atria and His bundle (through the $A V$ node). $A_{1}$ and $A_{6}$ were unable to activate the ventricles because the latter had already been depolarized by the impulses traversing the accessory pathway. The last three $Q R S$ complexes resulted from exclusive normal pathway conduction with block in the right bundle-branch. They were preceded by $H$ deflections with normal HV intervals. During this part of the tracing there was 2:I AV conduction through the normal pathway. None of the impulses propagated through the accessory pathway. The 2:I and 3:I pattern that (at the beginning of the tracing) had been occurring through the accessory pathway could no longer occur because $A_{10}, A_{12}$, and $A_{14}$ reached the ventricles slightly ahead of those impulses $\left(A_{12}\right.$ and $\left.A_{14}\right)$ which otherwise would have been totally responsible for the production of the $Q R S$ complexes.

AV node. Concealed conduction through the AV node produced enough $R$ to $R$ prolongation of consecutive narrow $Q R S$ complexes to allow conduction through the accessory pathway. On the other hand, when the effective refractory period of the accessory pathway was very short the atrial impulses reached the ventricles through the accessory pathway. Concealed conduction through this pathway resulted in long $R R$ cycles terminated either by anomalous, or normal, QRS complexes (depending on the presence of coexisting concealed conduction through the AV node).

A thorough evaluation of concealed conduction required the recording of His bundle electrograms and knowledge of the functional properties of both pathways. That the effective refractory period of the accessory pathway was relatively long in some cases, while being extremely short in others, has been attributed to differences in the physiological properties of the accessory pathway. It appears as if in the former instances this structure behaved (functionally) as Purkinje tissue, whereas in the latter it resembled ordinary atrial muscle. However, this assumption requires corroboration by further electrophysiological and anatomical studies.

Wide $Q R S$ complexes during atrial flutter and fibrillation in pre-excitation (WPW) syndrome

Intracardiac recordings were necessary to determine the nature of wide QRS complexes seen during the tachyarrhythmias. Whereas some beats were pure, or fusion, WPW complexes, others resulted from exclusive accessory pathway conduction with distortion created by propagation during the period of incomplete ventricular recovery. Reaching the correct diagnosis required knowledge of the QRS morphology produced by exclusive accessory pathway conduction (in which the forward $\mathrm{H}$ deflection was inscribed after the onset of ventricular depolarization) and of that resulting from exclusive normal AV pathway conduction with, or without, bundle-branch block in which the forward 
$\mathrm{H}$ deflection was inscribed at its normal time in front of the ventricular complexes.

\section{References}

Befeler, B., Castellanos, A., Jr., Castillo, C. A., Agha, A. S., Vagueiro, M. C., and Myerburg, R. (1973). Arrival of excitation at the right ventricular apical endocardium in Wolff-Parkinson-White syndrome type B. Circulation. In the press.

Castellanos, A., Jr., and Castillo, C. A. (1972). His bundle recordings in the right bundle-branch block coexisting with iatrogenic right ventricular pre-excitation. British Heart fournal, 34, 153.

Castellanos, A., Jr., Castillo, C. A., Agha, A. S., Befeler, B., and Myerburg, R. J. (1973). Functional properties of accessory AV pathways during premature atrial stimulation. British Heart fournal, 35, 578.

Castillo, C. A., and Castellanos, A., Jr. (1970). His bundle recordings in patients with reciprocating tachycardias and Wolff-Parkinson-White syndrome. Circulation, 42, 271.

Castillo, C. A., Castellanos, A., Jr., Befeler, B., Myerburg,
R. J., Agha, A. S., and Vagueiro, M. C. (1973). Arrival of excitation at right ventricular apical endocardium in Wolff-Parkinson-White syndrome type $A$, with and without right bundle-branch block. British Heart fournal, 35, 594.

Herrmann, G. R., Oates, J. R., Runge, T. M., and Hejtmancik, M. R. (1957). Paroxysmal pseudoventricular tachycardia and pseudoventricular fibrillation in patients with accelerated A-V conduction. American Heart fournal, 53, 254.

Katz, L. N. and Pick, A. (1956). Clinical Electrocardiography Part I. The Arrhythmias. Lea and Febiger, Philadelphia. Laham, J. (1969). Le Syndrome de Wolff-Parkinson-White, Actualités Électrocardiographiques. Maloine, Paris.

Scherf, D., and Cohen, J. (1964) The Atrioventricular Node and Selected Cardiac Arrhythmias. Grune and Stratton, New York.

Wit, A. L., Weiss, M. B., Berkowitz, W. D., Rosen, K. M., Steiner, C., and Damato, A. N. (1970). Patterns of atrioventricular conduction in the human heart. Circulation Research, 27, 345.

Request for reprints to Dr. A. Castellanos, Jackson Memorial Hospital, Miami, Florida, U.S.A. 